left upper arm. Of these, No. 1 was situated on the inner and anterior surface of the left elbow-joint, just above the inner condyle; it took a direction backwards and upwards. No. 2, bullet-wound; point of entrance on the outer side of middle of left upper arm. No. 3, bullet-wound (point of exit of No. 2) of inner side of left upper arm, just below axilla. 'The bullet which inflicted No. 1 wound had passed into the elbow-joint and fractured the lower end of humerus, dividing the inner from the outer condyle. On patient's arrival he was feverish and weak. Temperature $102^{\circ}$. These wounds were inflicted by Bulgars some eight days ago, since when he has had nothing applied to them except some old dry rags and finely-cut tobacco. I cleaned out the wounds, and well washed them with carbolic acid solution (1 to 30 ).

On August 6th, at noon, the patient having rested, I inserted some solution of morphia (equal to three-quarters of a grain) under the skin of the left pectoral muscle, and in ten minutes afterwards I began the inhalation of chloroform (simple). The patient almost at once passed under its influence. I then made an incision about three inches long, beginning it from just above the inner condyle and carrying it through the wound caused by the bullets. Then $I$ inserted a knife, and carefully divided the ligaments. A second incision I made on the outer aspect of the joint. Then I inserted the blade of Butcher's saw in front of the humerus, above the extent of the fracture. Having fixed on the frame of the saw, I cut through the bone from before backwards. I now easily removed the fragments, which were in a state of caries, and found the bullet lodged in the bone at the root of the inner condyle. Slight bleeding occurred from the divided anterior ulnar recurrent, but this was arrested by twisting. The cavity was now well syringed out with carbolic lotion ( 1 to 30 ), and a drainage-tube inserted, the ends hanging out of the joint, one on either side. Both wounds were stitched together with carbolised catout. Carbolised wool and gauze and paper were applied over both wounds. Patient was put to bed with the arm, resting on a soft pillow, bent to an angle of $55^{\circ}$ to $60^{\circ}$. He had no spasm and felt no pain of any kind during the operation, which lasted thirty-five minutes. He was sensible for the greater part of the operation, and answered correctly all questions put to him. He says he felt a sense of dragging in the arm during the operation.

8th.-Patient complaining of pain and heat of elbow. Removed all dressings. Slight purulent discharge. Injected wound, and through drainage-tube some solution of carbolic acid ( 1 to 30), then applied pads of lint, soaked in the same solution, over the wound. Fixed arm to the pillow with bandages. Applied constantly a solution of carbolic acid $(1$ to 40$)$ to the parts over wound.

20th.-Patient doing very well. Wound united except at entrance and exit of tube. Removed tube and syringed passage with carbolic solution (1 to 30 ). Again applied pads of lint, and ordered patient to keep them constantly moist with the solution. He rapidly recovered, but I kept him under observation lest he might displace the position of the joint, which I had secured by means of an angular splint applied along the inner side of arm and forearm.

On Nov. 12th I sent him home. The joint solid throughout, but with slight power of extension and flexion.

March 1st.-Patient visited me strong and well. Joint quite filled in ; in parts rather an overgrowth of bone. Has considerable power of flexion and extension. A partiallyformed false joint must have ensued in this case.

Kustendje, Bulgaria.

\section{A CASE OF}

THREE HARD MASSES OF COCOA-NUT FIBRE FOUND AFTER DEATH IN THE STOMACH OF A LUNATIC.

BY WILLIAM H. HIGGINS, M.B.,

ASISTANT MEDICAL OFFICER, LEICESTERSHIRE AND RUTLAND LUNATIC ASYLUM.

Is December, 1862, J. F- a male patient, aged thirtyfive, was admitted into this institution labouring under chronic mania of three years' duration. He died on the 11 th inst., and the autopsy seems to me worthy of being recorded, as I have nowhere seen mention made of a similar case.
On sectio abdominis the stomach appeared considerably enlarged, in situ, and on being opened, the walls were found much thickened, but free from disease; it contained no food, but three hard black masses of rounded form disclosed themselves to view, lying end to end along its greater curvature Together the three weighed thirty-seven ounces. The hypertrophied stomach alone, when empty, weighed eleven ounces. These masses, black without from the action of the acids of the stomach, and brown on section, present a fibrous appearance, and consist throughout of fibres of cocoanut matting intimately compressed and spirally rolled up into hard, firm, rounded bodies. No. l, the largest, lying in the greater end of the stomach, is six inches long, three inches thick, and weighs sixteen ounces and a half, resembles a thick roll, rounded at the end near the cardiac orifice, and truncated obliquely and slightly concave at the other end, where, it is contact with No. 2, a globular mass, the size of a large orange, flattened at the poles, three inches and a half in broad diameter and two inches in polar diameter, weighing eleven ounces and a half, evidently owing its globular form to rotation (by physiological action during digestion) between the ends of No. 1 ; and No. 3, a mass of irregular form, four inches and three-quarters long and two to three inches thick, weighing nine ounces, round at the end lying near the pylorus, concave where it touched, and, as it were, forming a shallow socket for the interme diate globular No. 2 to rotate against. The liver weighed thirty-five ounces, was universally ochrey in colour, and of nutmeggy appearance on section. The gall-cyst was normal; no calculi in it.

The disease that proved fatal was cancer (encephaloid and scirrhus) of the head of the pancreas, weighing with the latter twenty-two ounces, connected to surrounding parts. There was secondary cancer of mesenteric glands.

History of patient.-Wince he had been an inmate (i.e., for fifteen or sixteen years) he had been observed to have a constant habit of putting rubbish into his mouth, and of eating the fibres of the cocoa-nut matting laid down in the ward, shreds of mops, and the fluffy sweeping of the floor. He had ever an anæmic appearance, but did not complain used to work on the farm and in his ward, and never seemed to ail anything: Ate his food regularly, but during the last eight or ten years he was observed to sleep on his face, or to assume a posture on his arms and knees in bed, manifestly to relieve the pressure of the three masses upon the parts behind the stomach. About four months ago he was seized rather suddenly with a severe attack of indigestion, vomiting, and purging of clay-coloured stools, pain in the abdomen, \&c. Under treatment these icteric symptoms disappeared, and he was up and dressed for about one month. He soon became dropsical and sank. He had long evinced great impairment of mind; was reticent, and no reliable or pertinent replies could be elicited as to his sensations. During life the lumps were, from the icteric symptoms, attributed to enlarged liver.

Birkenhead.

\section{NOTE ON THE EXCISION OF THE PHALANGEAL JOINTS.}

BY ENGLEDUE PRIDEAUX, L.R.C.P.L, M.R.C.S.E., LATE RESIDENT SURGEON AT THE SCARBOROUGH DISPENSARY AND ACCIDENT HOSPITAL.

IT may be thought that the subject of this note is of very little importance ; but we should attach almost as much importance to the best modes of treating minor complaints as to those of treating major ones, and although the fingers are comparatively small parts of the human body, they are relatively very useful and important ones, and should not be neglected. A stiff finger to a labouring man, especially when engaged in certain occupations, often means inability to earn his livelihood, and after an injury to his finger almost necessitating amputation, when he begs to have it left, and makes light of its probable stiffness, that result often causes him to return to have it removed after all, as he finds it would be better to be without it than let it im. pede him in his daily labour. 